lich zu warnen, und stellte den liberalen Staaten die totalitären Systeme Deutschlands und der Sowjetunion gegenüber. ${ }^{239}$

Während des Krieges wurde Borkenau zwischenzeitlich als »feindlicher Ausländer« in Australien interniert. Nach Kriegsende kehrte er nach Deutschland zurück und wurde 1946 als Professor für Mittlere und Neuere Geschichte, besonders Geschichte der Sozialtheorien, an die Universität Marburg berufen. 1947 veröffentlichte er seine Antrittsvorlesung Drei Abhandlungen zur deutschen Geschichte. ${ }^{240}$ Mittels seiner Kontakte zu den amerikanischen Besatzungsbehörden war Borkenau maßgeblich an der Gründung der neuen wissenschaftlichen und politischen Zeitschrift Ostprobleme beteiligt, die er als Chefredakteur herausgab und die ab August 1949 erschien. Bis zu seinem Ausscheiden im März 1951 interessierte ihn insbesondere die laufende Entwicklung im kommunistischen Lager, die zu seinem letzten größeren Buch Der europäische Kommunismus führte. ${ }^{241}$ Hier entwickelte Borkenau eine völlig neuartige Methode der Interpretation: Die »Kreml-Astrologie« ging von der Annahme aus, dass sich die sowjetische Politik aus einem permanenten »Machtkampf rivalisierender Fraktionen und Führungsgruppen entwickelt, und dass es möglich sei, auf Grund sorgfältiger Analyse der offiziellen Dokumente die Zusammensetzung und politische Konzeption der kämpfenden Gruppen zu erschließen«. ${ }^{242}$ Für Löwenthal war es die Anwendung ebendieser Methode auf die Situation kurz vor Stalins Tod und danach, die Borkenaus publizistischen Ruf in den letzten Lebensjahren schnell anwachsen ließ. In den Jahren des Kalten Krieges drehten sich seine zahlreichen Zeitschriftenveröffentlichungen im Monat und diversen anderen Organen - in allererster Linie um die Analyse des sowjetischen Kommunismus. Daneben wurde die Auseinandersetzung mit den Geschichtsphilosophien von Karl Marx, Oswald Spengler und Arnold J. Toynbee zu einem weiteren Schwerpunktthema. ${ }^{243}$ Borkenau verstarb am 18. Mai 1957 im Alter von nur 56 Jahren in Zürich an einem Herzinfarkt.

\title{
4.2 Richard Löwenthal
}

Wenige Wochen, nachdem der Student Benno Ohnesorg im Verlauf der Demonstration gegen den Schahbesuch von einem Polizisten in Berlin erschossen wurde und das als Fanal für die außerparlamentarische Protestbewegung in der Bundesrepublik wirkte, traf der 59 Jahre alte Berliner Politikprofessor Richard Löwenthal im Rahmen einer vom Sozialistischen Deutschen Studentenbund initiierten, vom 10. bis zum 13. Juli 1967 dauernden Veranstaltungsreihe, im voll besetzten Audimax der Freien Universität Berlin, unter anderem auf Rudi Dutschke und Herbert Marcuse. In der Podiumsdiskussion zum Thema »Moral und Politik in der Überflußgesellschaft« sagte Löwenthal angesichts der Ausführungen von Marcuse, der mit seiner Studie Der ein-

239 Ders., The Totalitarian Enemy, London 1940.

240 Ders., Drei Abhandlungen zur deutschen Geschichte, Frankfurt a. M. 1947.

241 Ders., Der europäische Kommunismus. Seine Geschichte von 1917 bis zur Gegenwart, Bern 1952.

242 Richard Löwenthal, In memoriam Franz Borkenau, in: Der Monat 9 (1957), H. 106, S. 59 f. Siehe Franz Borkenau, Was ist Kreml-Astrologie? in: Der Monat 7 (1955), H. 79, S. 32-39.

243 Siehe bes. Franz Borkenau, Ende und Anfang. Von den Generationen der Hochkulturen und von der Entstehung des Abendlandes, Stuttgart 1984. 
dimensionale Mensch im selben Jahr ein theoretisches Schlüsselwerk der Studentenbewegung veröffentlichte und als eigentlicher Mentor der Revoltierenden galt:

Ich greife als erstes die Frage heraus, wo dieses System als Canzes erscheint. Als Ganzes erscheint es am ehesten in der Außenpolitik, und Marcuse hat gestern pointiert gesagt, dass dieses System immer wieder Kriege produziert, und er hat bei aller Kritik an dem ihm entgegengesetzten System des östlichen Totalitarismus hinzugefügt, dass dieser östliche Totalitarismus immerhin nicht expansiv und nicht aggressiv sei. Ich fand das kühn, uns das hier in Berlin zu erzählen. Wir haben hier die Expansion des östlichen Systems in den Jahren nach Hitlers Krieg ja alle miterlebt, und während es natürlich dem Einzelnen freisteht, diese Expansion als geschichtlich gerechtfertigt anzusehen, so ist es, glaube ich, nicht möglich, ihre Tatsache zu leugnen. ${ }^{244}$

Die hier expressis verbis in Richtung Marcuse zielende Kritik der Unterschätzung der aggressiven auf Expansion ausgerichteten Außenpolitik des »östliche[n] Totalitarismus«, sprich: des sowjetischen Kommunismus unter Stalin nach 1945, radikalisierte Löwenthal nur wenige Jahre später. Im Kontext einer theoretischen und politischen Auseinandersetzung war dieses Mal indessen die Neue Linke der Adressat seiner substanziellen Kritik. Nachdem die Rebellion ihren Höhepunkt schon lange überschritten hatte und einen immer mehr regressiven Verlauf nahm, veröffentlichte er 1970 den aus zwei Aufsätzen bestehenden Band Der romantische Rückfall. Beide Aufsätze wurden nicht zufällig in jeweils gekürzter Fassung im Monat vorab veröffentlicht. Vor dem Hintergrund der eigenen Erfahrungsgeschichte mit den totalitären Herrschaftssystemen des Nationalsozialismus und des Sowjetkommunismus sah sich der ehemalige Kommunist Löwenthal veranlasst, auf spezifische Gefahren aufmerksam zu machen. Einerseits warnte er im ersten Aufsatz die von der marxistischen Theorie inspirierten Intellektuellen der sogenannten Neuen Linken davor, nicht ein zweites Mal wie die »deutsche Intelligenz« in den 1920er- und 1930er-Jahren dem politischen und akademischen Konformismus anheimzufallen und mithin den - wie Löwenthal meinte - immer wieder kehrenden "Verrat« (Julien Benda) an den genuinen Aufgaben des kritischen Intellektuellen zu üben. ${ }^{245}$ Andererseits versuchte er im zweiten Aufsatz Unvernunft und Revolution ${ }^{246}$ im Kontext der Thematisierung der Genesis des neuen Typs revolutionärer Bewegung der Studenten in den westlichen Staaten der späten 1960er-Jahre im Allgemeinen und in der Bundesrepublik im Besonderen das Gefahrenpotenzial für die »westliche Zivilisation« zu diagnostizieren. Das Besondere der »irrationalen Revolte« der Neuen Linken - zu deren Opfer auch Löwenthal sowie sein Freund und Kollege Ernst Fraenkel von der Freien Universität Berlin wurden, als

244 Löwenthal auf der Podiumsdiskussion zum Thema »Moral und Politik in der Übergangsgesellschaft«; geleitet von Jacob Taubes mit Herbert Marcuse, Prof. Schwan, Prof. Claessens, Peter Furth, Rudi Dutschke und Wolfgang Lefèvre. Hier zit. n. Herbert Marcuse, Das Ende der Utopie. Vorträge und Diskussionen in Berlin 1967, Frankfurt a. M. 1980, S. 89.

245 Siehe hierzu das erste Kapitel »Zwischen Konformismus und Sezession« in: Richard Löwenthal, Der romantische Rückfall. Wege und Irrwege einer rückwärts gewendeten Revolution, Stuttgart u. a. 1971, S. 11-40, sowie den Vorabdruck in: Der Monat 20 (1968), H. 239, S. 32-38.

246 Siehe unter demselben Titel in: Löwenthal, Der romantische Rückfall, S. 41-87, sowie den Vorabdruck unter dem Titel Unvernunft und Revolution. Über die Loslösung der revolutionären Praxis von der marxistischen Theorie in: Der Monat 21 (1969), H. 251, S. 71-87. 
beide mit den von ihnen als »Terror« empfundenen Gewaltaktionen der Studenten konkret konfrontiert wurden und angesichts der neuerlichen totalitären Erfahrung in Deutschland beinahe zum zweiten Mal ins Exil gehen wollten -, bestand für ihn darin, dass sie eine Renaissance der romantischen ideologischen Tradition darstellte und mithin ein »Wiederaufleben der Gefühlshaltung« zur Folge hatte, das dazu führte, dass die Revoltierenden in ihrer »Verzweiflung« mittels der intellektuellen Bedeutung Marcuses $^{247}$ die moderne Industriegesellschaft in der westlich-kapitalistischen wie auch in der sowjetisch-kommunistischen Form infrage stellten. Mit Blick auf die Gefahren konstatierte der Autor eingedenk des Totalitarismus des 20. Jahrhunderts und vor dem Hintergrund, dass er durchaus eine »Verwandtschaft« zwischen der Neuen Linken und den $»$ Faschisten $« \operatorname{sah}^{248}$ :

»Sie besteht nicht in der Bildung eines >Blocks der Dritten Welt< draußen oder von Revolutionen im Innern, sondern in Zerstörung, Zerfall und Barbarisierung.« Und weiter:

Ebenso ist die wirkliche Bedrohung im Westen nicht, dass die jungen Extremisten »die Macht übernehmen«; sie können nicht einmal die Universitäten übernehmen. Aber sie können sie lähmen und in manchen Fällen zerstören, indem sie zuerst die Atmosphäre der Toleranz und der rationalen Diskussion zerstören, ohne die der Geist nicht atmen kann; [...] und sie können einen Cegenschlag von Polizeibrutalität und Rechtsextremismus hervorrufen, der ihnen objektiv helfen wird, das Funktionieren der Demokratie zu stören und die konstruktive Lösung dringender Fragen zu verhindern. ${ }^{249}$

Löwenthal wurde am 15. April 1908 als Sohn eines jüdischen Handelsvertreters und einer Schauspielerin in Berlin geboren. Nach dem Besuch des »Mommsen-Gymnasiums «egann er in Berlin mit dem Studium der Nationalökonomie und Soziologie und promovierte 1931 in Heidelberg (wo z. B. Max Weber und Karl Mannheim zu seinen Lehrern zählten) mit der Arbeit Die Marxsche Theorie des Krisenzyklus zum Dr. phil. Bereits in der Berliner Zeit seines Studiums arbeitete er im Kommunistischen Studentenverband und trat im Alter von 18 Jahren 1926 in die KPD ein. Insbesondere die Kontinuität der gesellschaftlichen und politischen Strukturen und die dominante Rolle der traditionellen Elite in der Weimarer Republik lehnte er ab. Im selben Jahr lernte er Borkenau kennen und wurde nicht nur dessen Nachfolger als kommunistischer Studentenführer, sondern seitdem wurde der führende Parteitheoretiker und spätere antitotalitäre Intellektuelle sowohl sein »politischer Lehrer« als auch (bis zu

247 Löwenthal, Der romantische Rückfall, S. 68.

248 Wortwörtlich schrieb der Autor hierzu: »Schließlich sind auch die heutigen Hochschulrebellen nicht die Vorläufer von politischen Revolutionen, wie einst die Studentenbewegungen im zaristischen Rußland, in der Weimarer Republik oder in Britisch-Indien: Sie entfalten sich nicht in stagnierenden oder politisch unterdrückten Gesellschaften und sind nicht der artikulierte Ausdruck der unartikulierten Stimmung breiter Volksmassen. Überdies sind die Studenten der >Neuen Linken trotz aller verwandten Züge, die wir erwähnt haben, keine Faschisten - und Bakunisten haben noch nie und nirgends die Macht erobert; sie würden in der Tat nicht wissen, was sie damit anfangen sollten." (Ebd., S. 85 f.)

249 Ebd., S. $86 \mathrm{f}$. 
dessen Tod) sein persönlicher Freund. ${ }^{250} 1929$ wandte sich Löwenthal gegen die stalinistische »Sozialfaschismus-These«, demzufolge die SPD und nicht die NSDAP der Hauptfeind der Kommunisten sei. Daraufhin schloss ihn die KPD als »Rechtsabweichler« im selben Jahr aus der Partei aus. Neben ehemaligen Parteiintellektuellen wie z. B. Karl Korsch zählte er danach zur »KPD-Opposition« und fungierte von 1932 bis 1933 als Mitarbeiter des theoretischen Parteiorgans Die Gesellschaft. Nach der nationalsozialistischen Machteroberung im Januar 1933 hatte er als exponierter »Ideologe« entscheidenden Einfluss am Aufbau des illegalen Elitekaders der sozialistischen (Widerstands-)Gruppe Neu Beginnen (NB). ${ }^{251}$

Fortan lebte Löwenthal im Untergrund. 1935 musste er Deutschland verlassen und ging über die Stationen Prag und Paris in das Londoner Exil, wo er unter seinem Decknamen Paul Sering neben zahlreichen politischen Publikationen auch seine persönliche Theorie des Faschismus in der vom führenden SPD-Ökonomietheoretiker Rudolf Hilferding herausgegebenen Zeitschrift für Sozialismus (ZfS) veröffentlichte, die beispielsweise die frühe Totalitarismusstudie von Borkenau beeinflusste. ${ }^{252}$ In klarer Abgrenzung gegen den von der Komintern proklamierten Totalitarismusbegriff bezeichnete für ihn der anfangs nur auf den Faschismus bezogene Begriff des »totalen Staates« schon zu diesem Zeitpunkt einen neuartigen Herrschaftstypus, der prinzipiell durch unterschiedliche »Klasseninhalte « realisiert wurde. ${ }^{253}$ Seine Analysen zur historischen Genesis des NS-Staates in Deutschland auf dem Boden der voll entwickelten Produktivkräfte des krisenhaften Kapitalismus - "mit Hilfe der alten reaktionären Mächte« (Sering) der Weimarer Republik -, die er in einigen Aufsätzen in der ZfS anfertigte, ${ }^{254}$ beeinflussten unter anderem den Behemoth von Neumann. Nach einem Forschungsstipendium 1936/37 in London - wo er auch seinen Freund Borkenau wieder traf - übernahm Löwenthal Aufgaben in der Prager Zentrale der Gruppe Neu Beginnen und reiste im April 1938 nach Paris. Hier arbeitete er unter seinem

250 Siehe die persönlichen Anmerkungen von Richard Löwenthal im »Vorwort zur amerikanischen Erstausgabe« in: Franz Borkenau, Ende und Anfang, S. 7-10, sowie Richard Löwenthal, In memoriam Franz Borkenau, in: Der Monat 9 (1957), H. 106, S. 57-60.

251 Die Gruppe »Neu Beginnen« wurde allerdings schon vor der »Machtergreifung« des Nationalsozialismus gegründet. Sie wurde vor dem Hintergrund der Krise der sozialistischen Arbeiterbewegung in Deutschland und insbesondere aufgrund der immer stärker werdenden politischen Kämpfe zwischen der SPD und der KPD sowie des damit einhergehenden Zerfalls der Arbeiterbewegung in »kleine Splittergruppen«ins Leben gerufen. Für die Gründung zeichnete eine kleine Gruppe um Walter Löwenheim verantwortlich, die größtenteils aus der Kommunistischen Partei ausgeschlossen wurden. Siehe Richard Löwenthal, Die Widerstandsgruppe »Neu-Beginnen«, Berlin 1982 (Beiträge zum Thema Widerstand 1933-1945 20), S. 3, sowie Biographisches Handbuch der deutschsprachigen Emigration nach 1933 (hg. vom Institut für Zeitgeschichte, München und von der Research Foundation for Jewish Immigration, Inc., New York; unter der Cesamtleitung von Werner Röder und Herbert A. Strauss). Bd. I: Politik, Wirtschaft, Öffentliches Leben, München 1999, S. 458.

252 Borkenau, The Totalitarian Enemy, S. 8.

253 Siehe Sering, Der Faschismus, S. $839 \mathrm{ff}$.

254 Das Buch Biographisches Handbuch der deutschsprachigen Emigration nach 1933 enthält folgende Hinweise auf Veröffentlichungen von Löwenthal unter dem Pseudonym bzw. seinem Decknamen »Paul Sering« in der Zeitschrift: Der Faschismus. System und Widersprüche, in: ZfS 26/27 (1935); Historische Voraussetzungen des deutschen Nationalsozialismus, in: ZfS 30 (1936) und Die Aufgaben der deutschen Revolution, in: ZfS 33 (1936), S. 458; vgl. auch ders., Die Wandlung des Kapitalismus, in: ZfS 20/21 (1935). 
Pseudonym im Redaktionsstab der von Münzenberg herausgegebenen Zeitschrift Die Zukunft mit. Nach erfolgter Annäherung an den sogenannten Austromarxismus Otto Bauers und an Grundpositionen der traditionellen linken SPD-Parteiopposition war er im darauf folgenden Jahr Mitverfasser der anonymen Schrift Der kommende Weltkrieg. Aufgaben und Ziele des deutschen Sozialismus, die den Versuch darstellte, eine linkssozialistische Positionsbestimmung angesichts des prognostizierten Krieges in Europa zu begründen. ${ }^{255}$

Mit der »NB-Auslandszentrale« übersiedelte Löwenthal im Sommer 1939 nach London und begann nach dem Kriegsausbruch in der Redaktion von Reports from Inside Germany und ab 1942 bei der Nachrichtenagentur Reuters zu arbeiten. Dazwischen propagierte er hinsichtlich der alliierten Kriegsziele im Oktober 1941 in Klare Fronten für eine »deutsche Revolution zwischen den Weltmächten «, ${ }^{256}$ die er allerdings 1943 aufgrund der sowjetischen Außenpolitik gegenüber Polen radikal revidierte. Fortan korrespondierten seine politischen Grundpositionen und Vorstellungen mit denen der westlichen Staaten. Speziell mit der britischen und der amerikanischen Arbeiterbewegung sah das seit 1945 eingeschriebene Mitglied der SPD Gemeinsamkeiten, sodass sich zum Beispiel zur Fabian Society eine enge Verbindung entwickelte; als Beiratsmitglied der International Socialist Forum plädierte Löwenthal für eine Rekonstruktion der II. Internationalen und sprach sich - beeinflusst durch das Nachkriegsprogramm der britischen Arbeiterbewegung - auch mit Blick auf Deutschland für die parlamentarische Demokratie auf der Basis einer staatlichen Investitionslenkung aus, die sich dadurch auszeichnen sollte, dass sie sich an den tatsächlichen Interessen der arbeitenden Bevölkerung orientiert. Auf dem theoretischen und politischen Boden der zurückliegenden Jahre entstand 1947 - dem Jahr, in dem Löwenthal die britische Staatsbürgerschaft erhielt - das Buch Jenseits des Kapitalismus, mit dem er sich in die Nachkriegsdiskussion um den >Dritten Wegく zwischen den beiden Supermächten einschaltete und sich schnell einen Namen machte. Dem Autor war es in der Untersuchung darum zu tun, der Öffentlichkeit den Entwurf einer Neubestimmung des »demokratischen Sozialismus im Zeitalter der Planung und der totalitären Katastrophe« zu präsentieren, und zwar, so Löwenthal, in Abgrenzung zum westlichen Kapitalismus (und den »rückwärtsgewandten Utopisten des wirtschaftlichen Liberalismus wie Hayek und Roepke«) sowie dem Sowjetkommunismus. ${ }^{257}$

255 Der kommende Weltkrieg. Aufgaben und Ziele des deutschen Sozialismus. Eine Diskussionsgrundlage, Paris 1939 (Verfasser der anonymen Schrift waren Paul Sering, Karl Frank, Joseph Buttinger und Josef Podlipnig).

256 Hierzu steht im Buch Biographisches Handbuch der deutschsprachigen Emigration nach 1933, dass in diesem Fall Löwenthal und andere Mitstreiter von »Neu-Beginnen «aufgrund der konzeptionellen Ziele eines demokratischen sozialistischen Deutschlands in den »Grenzen der Weimarer Republik« als Vertreter eines »pangermanischen Denkens «angegriffen wurden (S. 458). Löwenthal vertrat in Klare Fronten nach dem deutschen Überfall auf die Sowjetunion und dem »Wiederaufleben einer \Ostorientierung ‘ der linken Exilgruppen eine Beteiligung der UdSSR an der Neuordnung Europas, um die Unterdrückung der revol[utionären] Bewegungen $\mathrm{u}$ [nd] die Oktroyierung der kapitalist[ischen] Gesellschaftsordnung (auch auf die UdSSR) durch die Westmächte zu verhindern«.

257 Paul Sering, Jenseits des Kapitalismus. Ein Beitrag zur sozialistischen Neuorientierung, Lauf bei Nürnberg 1947; siehe hierzu die Rezension des Buches unter dem Titel Jenseits des Sozialismus von Paul Kecskemeti, in: Der Monat 1 (1948), H. 3, S. 103-106, sowie die »Erwiderung« unter der Zeitschriftenrubrik »Briefe des Monat« von Löwenthal unter seinem Pseudonym - der Beitrag stand 
Nach seiner Rückkehr nach Deutschland 1948 war Löwenthal von 1949 bis 1955 für Reuters als Korrespondent in Westdeutschland tätig und arbeitete von 1954 an als außenpolitischer Leitartikler des Londoner Observer. In diesem Kontext begann zudem seine Mitarbeit für den in Berlin herausgegebenen Monat, wo er alsbald - mit letztlich mehr als 40 Veröffentlichungen - zu einem der gefragtesten und produktivsten Autoren reüssierte. Nach seinem ersten Artikel - der bereits genannten »Erwiderung« anlässlich der Rezension seines Buches Jenseits des Kapitalismus -, einem bald darauf erschienenen Beitrag im Rahmen des Symposiums »Erwacht Deutschland schon wieder? Der neue deutsche Nationalismus und seine Gefahren $\aleph^{258}$ und Aufsätzen zur politischen Situation in Frankreich und in Italien, ${ }^{259}$ über die Zustände an der Saar ${ }^{260}$ sowie zum Problem der westeuropäischen Wirtschaftseinheit ${ }^{261}$ hatte Löwenthal beim Berliner »Kongreß für kulturelle Freiheit« im Juni 1950 einen richtungsweisenden öffentlichen Auftritt. Hierbei führte er die lebensgeschichtlich aufgeladene, vehemente politische und analytische Auseinandersetzung mit der Sowjetunion primär unter Stalin ${ }^{262}$ auch

nach Information der Redaktion im Kontext der »Sozialismusdiskussion « im gleichen Heft - mit der Überschrift »Planung und Freiheit«, in: Der Monat 1 (1949), H. 5, S. 106-109. - Zu seinen zentralen Einsichten schrieb Löwenthal am Anfang dieser »Erwiderung«: »Die Frage nach der Vereinbarkeit von wirtschaftlicher Planung mit persönlicher und politischer Freiheit ist nach meiner Überzeugung die Grundfrage nach der Zukunft unserer Zivilisation. Die rückwärtsgewandten Utopisten des wirtschaftlichen Liberalismus wie Hayek und Roepke verneinen sie - und trösten sich mit der Versicherung, die universelle Entwicklung zur wirtschaftlichen Planung sei ein Irrweg, weder notwendig noch irreversibel. Die macchiavellistischen Propheten des bürokratischen Zeitalters wie Burnham verneinen sie - und prophezeien fatumgläubig den Sieg des Sklavenstaates in der jeweils aktuellsten Form. Die Bejahung der Frage macht die Grundthese meines Buches Jenseits des Kapitalismus aus; ihre Begründung, gestützt auf eine Analyse der Entwicklung der letzten Jahrzehnte, seinen gesamten Inhalt. «Und weiter hieß es: »lch suche darin zu zeigen, warum die zentrale staatliche Entscheidung über Umfang und Richtung der Investitionen in allen entwickelten Industriestaaten unvermeidlich geworden ist, und wie ihr der Prozeß der Ersetzung von Marktbeziehungen durch shierarchische` Beziehungen der Kooperation, Über- und Unterordnung zugrunde liegt. Ich untersuche die verschiedenen Planungsformen der gegenwärtigen Übergangszeit - kapitalistische und nichtkapitalistische im Hinblick auf die wirtschaftliche Machtverteilung; Wohlfahrtsplanung und Kriegsplanung im Hinblick auf den Zweck; demokratische und totalitäre im Hinblick auf die politische Form. Ich komme zu dem Ergebnis, daß es auf nichtkapitalistischer Basis, auf der Grundlage der Beseitigung des Monopoleigentums an den entscheidenden Industrien, grundsätzlich möglich ist, die zentrale Planentscheidung demokratisch, d. h. in freier Wahl zwischen alternativen Plänen, zu fällen, und ihre Durchführung auf allen Stufen demokratisch zu kontrollieren - sofern freiheitliche Institutionen, insbesondere Freiheit der Parteienbildung bestehen. In einem solchen politischen Rahmen, so behaupte ich, gibt die moderne Ökonomie die Möglichkeit, die Planung nicht zum Werkzeug einer selbstherrlichen Bürokratie, sondern zum Mittel der Hebung der allgemeinen Wohlfahrt und der schließlichen Überwindung der Klassenscheidung werden zu lassen - zum Mittel also einer im vollen Sinne des Wortes sozialistischen Entwicklung.«(S. 106)

258 Drei Wege deutscher Aussenpolitik, in: Der Monat 1 (1949), H. 8/9, S. 22-28.

259 Secessio Plebis, in: Der Monat 3 (1951), H. 36, S. 578-587.

260 Brief aus Saarbrücken: Zankapfel oder Bindeglied? Notizen von einer Reise ins Saargebiet, in: Der Monat 4 (1952), H. 44, S. 145-154.

261 Vom Sinn und Unsinn der Wirtschaftseinheit, in: Der Monat 4 (1952), H. 47, S. 451-467.

262 So schrieb Oliver Schmidt angesichts der persönlichen Erfahrungen Löwenthals mit dem Sowjetkommunismus und mit Blick auf das Ende des Zweiten Weltkrieges: „Sein bisheriges Leitbild verblasste, die Moskauer Prozesse Mitte der dreißiger Jahre und der Pakt Stalins mit Hitler-Deutsch- 
und vor allem vor dem Hintergrund der historischen Tatsache, dass die totalitären Herrschaftssysteme auf dem Boden der Krise der westlichen Gesellschaft entstanden sind. Nicht zuletzt deshalb schloss Löwenthal auch in Zukunft für die westliche Zivilisation die Möglichkeit einer (prä-)totalitären Entwicklung nicht aus: einerseits durch eine diplomatische und militärische Unterschätzung des imperialistischen Sowjetkommunismus und andererseits durch ein essenzielles innenpolitisches Problem des Westens; falls nämlich die demokratischen Gesellschaften angesichts der »Krise des Westens« sich als unfähig erweisen sollten, »konstruktive Lösungen für die inneren Probleme zu finden, und mithin beim Ziel der »Verteidigung der Freiheit« gewissermaßen versagten. ${ }^{263}$

Der Schwerpunkt der Veröffentlichungen von Löwenthal im Monat lag indes in erster Linie bei der Analyse des Kommunismus: nämlich des jugoslawischen Staates unter Tito im Allgemeinen ${ }^{264}$ und des stalinistischen Herrschaftssystems im Besonderen. Hierbei fokussierte er die facettenreiche politische, ökonomische, strukturelle und ideologische Entwicklung in der Sowjetunion vor ${ }^{265}$ und insbesondere nach dem Tode Stalins am 5. März $1953^{266}$ sowie der Geheimrede Chruschtschows anlässlich des XX. Parteitages der KPdSU im Februar 1956 vor dem Hintergrund der nachlassenden Rolle des Terrors ${ }^{267}$ und einer zeitgemäßen kommunistischen Machtideologie ${ }^{268}$ und -politik ${ }^{269}$. Des Weiteren beleuchtete der Renegat das Phänomen des Totalitarismus

land wirkten als Trauma lebenslang nach.« Und über seine Meinung zu Stalin zitierte er Löwenthal, der gegenüber dem Literaturwissenschaftler sagte: »Wo Stalin hintritt, wächst kein Gras mehr.«Siehe Oliver Schmidt, Jenseits der Ideologien. Vor zehn Jahren starb der Politologe Richard Löwenthal, in: Tagesspiegel vom 9. August 2001.

263 Löwenthal, Die Freiheit und der Konflikt der Weltmächte (Zitat S. 445).

264 Siehe hierzu folgende Beiträge: Brief aus Belgrad: Titos `Großes Experiment‘, in: Der Monat 5 (1952), H. 49, S. 39-48; Brief aus Belgrad: Modellfall Jugoslawien. Von der Kolchose zur Cenossenschaft, in: Der Monat 6 (1953), H. 62, S. 125-134. Vgl. auch zum sogenannten Fall des aus Jugoslawien stammenden kommunistischen Renegaten Milovan Djilas die Beiträge: Ketzer oder Pionier?, in: Der Monat 6 (1954), H. 67, S. 89-91, sowie Brief aus Belgrad: Führt Titos Weg zurück? Djilas und das jugoslawische Dilemma, in: Der Monat 10 (1957), H. 109, S. 30-37.

265 Siehe den bemerkenswerter- und zufälligerweise im Märzheft 1953 publizierten Beitrag: Der Hintergrund der Säuberungen, in: Der Monat 5 (1953), H. 54, S. 672-676.

266 Siehe (zum 5. März 1953) Stalins Vermächtnis. Zur Interpretation seiner letzten Schrift, in: Der Monat 5 (1953), H. 55, S. 16-25; Am Ende einer Epoche. Die Umwälzungen im russischen Großreich, in: Der Monat 5 (1953), H. 60, S. 572-580; Richard Löwenthal schreibt aus Belgrad: Die Zähmung der Ceheimpolizei, in: Der Monat 7 (1955), H. 77, S. 467-471; ders. schreibt aus London: Das russische Dilemma, in: Der Monat 7 (1955), H. 78, S. 561-565. Siehe auch den zehn Jahre nach Stalins Tod veröffentlichten historisch-analytisch sowie -politisch resümierenden Charakter besitzenden Beitrag: Chruschtschows unfertiger Staatsstreich. Vom Preis der Permanenz der Parteiherrschaft, in: Der Monat 15 (1963), H. 174, S. 7-14.

267 Siehe vor allem: Jenseits des Stalinismus, in: Der Monat 8 (1956), H. 91, S. 3-10; Richard Lowenthal [sic!] schreibt aus London: Verspäteter Thermidor?, in: Der Monat 8 (1956), H. 92, S. 80-84; Die letzte Säule, in: Der Monat 9 (1957), H. 107, S. 3-12.

268 Siehe ganz bes.: Ideologie und Realpolitik. Glauben die Kreml-Machthaber an Dogmen?, in: Der Monat 10 (1958), H. 117, S. 37-46.

269 Siehe zum Beispiel: Der verwandelte Gegner. Sowjetische Politik im Zeitalter des atomaren Patt, in: Der Monat 13 (1961), H. 148, S. 18-27; Der gescheiterte Durchbruch. Chruschtschows Vielfrontenkampf, in: Der Monat 15 (1962), H. 171, S. 9-18. 
unter anderem in folgender Hinsicht: als einen in der Geschichte neuartigen, totalitären Herrschaftstyp mit noch nie dagewesenen Massenverbrechen, der gegen die moderne Demokratie und deren substanzielle Freiheitsrechte ausgerichtet war; ${ }^{270} \mathrm{zu}-$ dem in dem Aufsatz Totalitäre und Demokratische Revolution, der hinsichtlich der wissenschaftlich-politischen Auseinandersetzung über die analytische, d.h. erkenntnistheoretische Reichweite der »klassischen « Totalitarismustheorie, ${ }^{271}$ für die Forschung eine überragende Bedeutung besaß. ${ }^{272}$

Neben der Veröffentlichung der Reuter-Biografie 1957 zusammen mit seinem Freund und »langjährigen politischen Weggefährten « Willy Brandt ${ }^{273}$ standen insbesondere die gesamten 1950er-Jahre für Löwenthal im Zeichen von Arbeiten mit dem Anspruch, die Entwicklung des sowjetischen und internationalen Kommunismus zu beschreiben und zu analysieren. Diese erschienen im Monat und in führenden internationalen fachlichen sowie politischen Zeitschriften ${ }^{274}$ und schlugen sich in der Studie Chruschtschow und der Weltkommunismus nieder. ${ }^{275}$ Zudem begann am Ende dieses Jahrzehnts seine akademische Laufbahn. Nach einer Tätigkeit als Gastdozent am Otto-Suhr-Institut an der Freien Universität Berlin 1959 und einer anschließenden Forschungsaktivität bis in das Jahr 1960 hinein am Russian Research Center der Harvard University in den USA wurde Löwenthal 1961 zum Ordinarius für Politikwissenschaft und für Geschichte und Theorie der Auswärtigen Politik am Otto-Suhr-Institut (OSI) berufen - zudem gleichzeitig zum Direktor der Sektion für Osteuropäische Zeitgeschichte am Osteuropa-Institut am selben Ort - wo er zum Beispiel in den folgenden Jahren als soziologischer und politologischer »Lehrer« wichtige Vertreter der (Westberliner) Studentenbewegung beeinflusste. ${ }^{276}$ Neben weiteren Aktivitäten

270 Siehe speziell Die Hölle auf Erden. Despotie im zwanzigsten Jahrhundert, in: Der Monat 9 (1957), H. 105, S. 3-8; vgl. auch im Rahmen der politischen und theoretischen Diskussion unter dem Titel Militärdiktatur und Demokratie. Drei Stimmen zu F. R. Allemanns Aufsatz über den »Staatsstreich als Regulativ« aus Anlass einer Analyse von Fritz René Allemann im Monat den Beitrag von Löwenthal, in: Der Monat 13 (1960), H. 145, S. 25-29.

271 Richard Löwenthal, Totalitäre und Demokratische Revolution, in: Der Monat 13 (1960), H. 146, S. 2940. Vgl. hierzu auch ders., Entwicklung kontra Utopie. Das kommunistische Dilemma, in: Der Monat 22 (1970), H. 266, S. 60-84.

272 Mitteilung von Martin Jänicke an den Verfasser in einem Gespräch in Berlin im November 1999.

273 Willy Brandt/Richard Löwenthal, Ernst Reuter. Eine politische Biographie, München 1957. Siehe den Auszug aus dem Buch, Willy Brandt/Richard Löwenthal, Die Berliner Blockade. Aus einer politischen Biographie Ernst Reuters, in: Der Monat 9 (1957), H. 108, S. 54-69.

274 Zum Beispiel in den »Schwesterzeitschriften«des Monat wie Encounter, Preuves oder Tempo Presente; zudem in den renommierten Zeitschriften wie Twentieth Century, Soviet Survey, Yearbook of World Affairs und China Quarterly (London), St. Antony's Papers (Oxford) sowie Commentary und Problems of Communism (Washington).

275 Richard Löwenthal, Chruschtschow und der Weltkommunismus, Stuttgart 1963. Vgl. hierzu die Rezension unter dem Titel Kommunismus im Zerfall von al [sic!], in: Der Monat 16 (1963), H. 181, S. 95 f. Das Buch hatte für die allgemeine Totalitarismusforschung einen nicht unerheblichen Einfluss und wurde u. a. von Arendt ausdrücklich gelobt. Siehe Hannah Arendt/Karl Jaspers, Briefwechsel 1926-1969, München 1993, S. 613, sowie Arendt, Elemente und Ursprünge totaler Herrschaft, S. 486.

276 Mit seinen ebenfalls als Totalitarismusexperten in Erscheinung tretenden Kollegen Otto Stammer und Hans-Joachim Lieber hatte Löwenthal starken Einfluß auf zum Beispiel Rudi Dutschke und Bernd Rabehl, also zwei Vertreter der Neuen Linken bzw. des SDS, die sowohl als revolutionäre (Ce- 
in Forschungseinrichtungen ${ }^{277}$ in den Vereinigten Staaten und in der Bundesrepublik fungierte Löwenthal über viele Jahre als wichtiger Berater der SPD-Parteiführung. Hierbei standen Fragen der Beziehung der SPD zu den Kommunisten im Vordergrund. Darüber hinaus spielte er eine zentrale Rolle im Kontext der »68er-Linken«, denen er als ehemaliger kommunistischer Intellektueller anfangs seine Diskussionsbereitschaft keineswegs verweigerte. Trotzdem lehnte der Berliner Politikprofessor die Ideen des SDS, Formen der genuinen "plebiszitären Demokratie« in der Gesellschaft als auch an den Universitäten einzuführen, strikt ab. Indes: Die öffentliche und radikale Distanzierung des durch die beiden »Großtotalitarismen« lebensgeschichtlich geprägten Löwenthal setzte erst zu dem Zeitpunkt ein, als er und Fraenkel mit den repressiven Methoden von Teilen der Berliner Linken konkrete Erfahrung machte und deren (semi-)totalitären Potenziale thematisierte und vor diesem Hintergrund den »konservativen Professoren-Kampfverband« Bund Freiheit der Wissenschaft mit ins Leben rief. ${ }^{278}$

Nach der Emeritierung im Jahre 1975, Aktivitäten und kritischen Einlassungen zur Politik der SPD veröffentlichte Löwenthal zudem Bücher, in denen er seinen Ruf als politischer Intellektueller und kritischer Diagnostiker der westlichen Demokratien unter Beweis stellte. ${ }^{279}$ Löwenthal verstarb am 9. August 1991 nach längerer Krankheit in einem Berliner Krankenhaus.

\subsection{Arthur Koestler}

Nachdem Arthur Koestlers Roman Darkness at Noon ${ }^{280}$ über die stalinistischen Säuberungen in der Sowjetunion in den Jahren 1936 bis 1938 nach der Veröffentlichung im Jahre 1940 für nur geringes Aufsehen sorgte, änderte sich dies mit der französischen Übersetzung nach dem Kriegsende. Paradoxerweise war an dem Erfolg des unter dem Titel Le Zéro et I'Infini 1946 in Frankreich erschienenen Buches, das innerhalb kürzester Zeit eine Auflagenhöhe von mehr als 400000 Exemplaren erreichte und somit alle Verkaufsrekorde des französischen Vorkriegsbuchhandels übertraf, die KPF wesentlich mitverantwortlich. Wie der Autor im Nachwort des Romans berichtete, kaufte die Kommunistische Partei, nachdem zum Beispiel der Versuch, den Verleger einzuschüchtern, misslang, ganze Lagerbestände vorstädtischer sowie provinzieller Buch-

sellschafts-)Theoretiker als auch als außerparlamentarische Politiker an der Konstituierung der Studentenbewegung in der Bundesrepublik maßgeblich beteiligt waren.

277 Siehe im Einzelnen hierzu Biographisches Handbuch der deutschsprachigen Emigration nach 1933 (S. 458).

278 Der Vollständigkeit halber soll hier nicht unerwähnt bleiben, dass Löwenthal den BFdW zu dem Zeitpunkt wieder verließ, als dieser Ende der 1970er-Jahre mehr und mehr in das politische Fahrwasser der CDU im Zusammenhang mit deren Bildungspolitik geriet.

279 Siehe exemplarisch Prolog: Dauer und Verwandlung, in: Richard Löwenthal/Hans-Peter Schwarz (Hg.), Die Zweite Republik. 25 Jahre Bundesrepublik Deutschland-eine Bilanz, 2. Aufl., StuttgartDegerloch 1974, S. 9-24; vgl. auch Vom kalten Krieg zur Ostpolitik, in: Ebd., S. 604-699. Siehe zudem Richard Löwenthal, Cesellschaftswandel und Kulturkrise. Zukunftsprobleme der westlichen Demokratien, Frankfurt a. M. 1979.

280 Darkness at Noon, London 1940 (dt. Ausg.: Sonnenfinsternis, Stuttgart 1948). 